\title{
Electrophysiological impact of diuretics in heart failure
}

Liv Storstein

The introduction of diuretics revolutionised treatment of acute and chronic heart failure by considerably reducing its excessive water load. The doses of mercurial diuretics, which were introduced first, had to be carefully calculated because of the danger of mercury intoxication. The thiazides greatly facilitated diuretic treatment as did the more powerful loop diuretics. Both these classes of diuretics induce hypokalaemia and hypomagnesaemia. Potassium sparing diuretics minimise this problem.

Heart failure is the final stage of various cardiovascular diseases: congenital heart disease, valvar heart disease, cardiomyopathies, hypertension, and coronary heart disease. In developing countries congenital and valvar heart disease have the highest prevalence, whereas in the western world hypertension and coronary heart disease are more prevalent. Arrhythmias and heart failure are closely associated. Sudden death occurs in $30 \%-50 \%$ of cases. ${ }^{1}$ Arrhythmias show an increasing prevalence with deteriorating pump function. ${ }^{2}$ Patients in New York Heart Association class IV thus have the highest prevalence of malignant ventricular arrhythmias and the highest mortality. The percentage of patients with moderate heart failure who die suddenly is high and highest in those with the most advanced pump failure. The electrophysiological impact of diuretics in patients with heart failure is therefore crucial.

There is little information on a possible direct effect of the various classes of diuretics on conduction time and arrhythmogenicity. Diuretics may influence the arrhythmias of heart failure through two different mechanisms: by influencing the evolution of heart failure through an effect on the underlying disease; and by inducing electrolytic disturbances, mainly hypokalaemia and hypomagnesaemia, that may increase and worsen arrhythmias in heart failure.

The main indications for diuretics are hypertension and heart failure. ${ }^{3}$ How then do diuretics influence hypertensive heart disease? Do diuretics effect the development of left ventricular hypertrophy, the first step in the development of heart failure in patients with hypertension? Left ventricular hypertrophy has been identified as a main independent risk factor for cardiovascular morbidity and is often associated with coronary artery disease in patients with hypertension, so the development of heart failure may be due to both left ventricular hypertrophy and coronary artery disease. Regression of left ventricular hyper- trophy is used as a criterion for a positive effect of treatment in patients with hypertension. In a recent review, Massie has criticised several existing studies, mainly due to design features. ${ }^{4} \mathrm{He}$ advocates the use of blinded design and randomised groups treated with placebo or active treatment for at least six months. Patients should have had symptoms of left ventricular hypertrophy with no coexisting cardiovascular disease and be untreated or without treatment for more than two months. The echocardiograms should be of high quality and readers blinded for treatment and sequence.

The Veterans Administration Cooperative Study provided the first conclusive evidence that antihypertensive treatment could reduce cardiovascular morbidity in patients with mild and moderate hypertension. ${ }^{5}$ Treatment consisted of a placebo or a combination of hydrochlorothiazide and reserpine, with added hydralazine if needed. Follow up was from 2.9 to five years. The study considered electrocardiographic criteria for left ventricular hypertrophy in patients showing voltage abnormalities, and $74.4 \%$ of treated patients reverted to normal voltage compared with $24 \%$ in the control group; the difference was highly significant with a $P$ value $<0.005$. This study thus showed that a treatment including diuretics could reduce electrocardiographic signs of left ventricular hypertrophy. The hypertension detection and follow up programme showed that patients with left ventricular hypertrophy had a three times higher five year mortality than those with a normal electrocardiogram. ${ }^{6}$ Most of the aggressively treated group was given diuretics alone or diuretics plus reserpine. Of these patients with electrocardiographic evidence of left ventricular hypertrophy, $54.3 \%$ reverted to normal or improved compared with $42.9 \%$ in the conservatively treated group. The multiple risk factor intervention trial also found fewer new cases of electrocardiographic left ventricular hypertrophy in the more aggressively treated group. ${ }^{7}$ The European Working Party on high blood pressure in the elderly showed an increase in electrocardiographic voltage in patients given placebos and a decrease in treated patients, $70 \%$ of whom received diuretics alone; the rest had added methyldopa. ${ }^{8}$ Massie $^{4}$ reviewed eight studies ${ }^{9-16}$ in which reduction in left ventricular hypertrophy was found by echocardiography and five studies $^{17-21}$ that could not show such changes. He suggested that the echocardiographic data are variable, but that studies that were better 
designed, longer in duration, included previously untreated or uncontrolled patients, and required blood pressure control, showed a strong tendency towards regression of left ventricular hypertrophy with diuretic treatment. The positive studies more often used chlorthalidone or indapamide rather than hydrochlorothiazide. Should these conclusions be validated in better designed trials, it seems likely that diuretic treatment may halt the development of congestive heart failure in hypertensive patients.

Diuretics are now the first line of treatment in heart failure, and the essence of their therapeutic value is that they correct the resulting venous overload of the failing heart by contraction of the intravascular and extracellular fluid volume by enhancing the renal excretion of saline. ${ }^{3}$ This primary effect is documented by the ability of diuretics to increase vascular compliance. This is achieved by two means: by reducing the saline content of the vessel walls; and also by liberation of vasodilator prostaglandins in vascular smooth muscle. Despite the widespread use of diuretics in the treatment of both acute and chronic heart failure, their symptomatic effects in both conditions are poorly described and their influence on prognosis is totally unknown. Despite the paucity of formal studies the diuretic group of drugs remain a mainstay in the treatment of both acute and chronic heart failure due to their effective relief of symptoms, advantageous pharmacodynamic activity, their relatively high benefit to risk ratio, and their low socioeconomic cost to the community. This last factor will be of increasing importance in the future as total health costs are exploding in industrialised countries. Drugs currently used in the treatment of heart failure show a 33-fold variation in the cost of a defined daily dosage. It becomes increasingly important to investigate the less expensive alternatives to find out if they are less, equal to, or more beneficial in the treatment of various stages of heart failure.

Diuretics have several known side effects: dehydration, electrolytic disturbances, disturbed renal function, deleterious change in lipid profile, induction of diabetes, impaired glucose tolerance, insulin resistance, and hyperuricaemia. ${ }^{22-25}$ Of special interest to the question of the electrophysiological impact of diuretics in heart failure, is their influence on potassium and magnesium. ${ }^{26-34}$ Both hypokalaemia and hypomagnesaemia are important risk factors for arrhythmias in heart failure. A reported meta-analysis by Collins et al of 14 randomised trials of antihypertensive drugs, involving 37000 patients who were treated for a few years, found that a reduction of 5-6 $\mathrm{mm} \mathrm{Hg}$ in diastolic blood pressure produced a $42 \%$ reduction in the incidence of stroke and a $14 \%$ reduction in the incidence of coronary heart disease. ${ }^{35}$ Most patients in these trials were treated with a regimen based predominantly on diuretics. The use of diuretics as first line treatment of hypertension has been challenged in recent years by the availability of vasodilators including cal- cium blockers and angiotensin converting enzyme (ACE) inhibitors. The potential deleterious effect of diuretics on potassium and magnesium concentrations and their possible proarrhythmic effect has been considered. This discussion was partly triggered by results from the multiple risk factor intervention trial (MRFIT) ${ }^{36} \mathrm{~A}$ subgroup analysis of this study suggested that men with previous electrocardiographic abnormalities at baseline might have an increased risk of sudden death when treated intensively with diuretics. A similar reanalysis performed by the hypertension detection and follow up programme (HDFP) ${ }^{37}$ failed to confirm the MRFIT findings. The relation between the use of diuretics, hypokalaemia; and cardiac arrhythmias has been investigated. Papademetriou et al repeatedly reported no increase in ventricular premature contractions with ambulatory electrocardiographic monitoring, ${ }^{30-33}$ although other investigators have found such a relation. The thiazide induced hypokalaemia is dose dependent and the use of lower doses may minimise the risk of arrhythmias.

Hypokalaemia has a clear effect on the electrophysiological properties of the heart by increasing resting membrane potentials and the duration of the action potential and refractory period. It is thus conducive to the genesis of re-entrant arrhythmias and by increasing threshold potential, also increases automaticity. Conductivity is decreased by hypokalaemia. This is reflected in a study by Steward and associates who performed a controlled prospective crossover study of patients with mild hypertension and ischaemic heart disease. ${ }^{38}$ During the potassium losing phase they found an increased frequency of ventricular premature contractions, a higher Lown grade during ambulatory electrocardiographic monitoring, prolonged duration, decreased phase zero velocity of the monophasic action potential, a prolonged ventricular effective refractory period, and an increased myocardial electrical instability as assessed by programmed ventricular stimulation. Hollifield and Slaton investigated the effect of increasing doses of hydrochlorothiazide $(12.5 \mathrm{mg}$ to $50 \mathrm{mg}$ daily in four steps) on serum potassium and magnesium concentrations and arrhythmias at rest and during static and dynamic exercises. ${ }^{39-41}$ In 38 patients who had previously experienced hypokalaemia, palpitation, or cardiac arrhythmias, they found an increased occurrence of premature ventricular contraction that significantly correlated with a decrease in serum potassium $(r=0.73$, $\mathrm{P}<0.001)$ and serum magnesium $(r=0.68$, $\mathrm{P}<0.001)$ and the product of the change of the two cations $(r=0.81, \mathrm{P}<0.001)$.

A recent critical review of Singh, et al discussed the relations of diuretics to potassium and magnesium deficiency, drug-induced long QT interval, cardiac arrhythmias, and sudden death. ${ }^{26}$ In opposition to the review by Freis $^{42}$ these authors conclude that the depletion of body potassium content in connection with hypokalaemia may be important in the genesis of arrhythmias and sudden death that 
are often seen in cardiac failure. They referred to positive results in trials of treatment for hypertension in which potassium conserving agents have been included as in the European Working Party on high blood pressure in the elderly trial, ${ }^{43}$ the Swedish trial in old patients with hypertension (STOP), ${ }^{44}$ and systolic hypertension in elderly patients (SHEP) trials. ${ }^{45}$

We can thus conclude that knowledge of the direct electrophysiological effects of diuretics is scarce. Diuretics may have proarrhythmic effects through induced electrolytic disturbances and possibly through other side effects. Diuretics may be indirectly antiarrhythmic through a positive influence on the underlying heart disease. Properly designed clinical trials are urgently needed to clarify the safety and the electrophysiological impact of diuretics in patients with heart failure.

1 Meinertz $T$, Hofmann $T$, Zehender $M$, Drexler $H$, Hohnloser $S$, Just $H$. $\beta$-blocking agents vs. antiarrhythmic interventions in heart failure complicated by
arrhythmias. $₹$ Cardiovasc Pharmacol 1990;16(suppl 5): S151-7.

2 Meinertz T, Hofmann T, Hohnloser SH, Zehender M, Just $H$. Arrhythmias and left ventricular dysfunction. fournal of Ambulatory Monitoring 1989;2:65-73.

3 Taylor SH, Storstein L. Diuretics in the treatment of heart failure. Herz 1983;8:11-22.

4 Massie BM. Effect of diuretic therapy on hypertensive left ventricular hypertrophy. Eur Heart $\mathcal{f} 1992 ; 13$ (suppl G) 53-60.

5 Pobleto PF, Kyle MC, Pipberger HV, et al. Effect of treatment on morbidity in hypertension: veterans administration cooperative 'study on antihypertensive agents: effect on the electrocardiogram. Circulation 1973;48:481-90.

6 Hypertension Detection and Follow-up Program Cooperative Group. Five-year findings of the hypertension detection and follow-up program. Prevention and reversal of left ventricular hypertrophy with antihypertensive therapy. Hypertension 1985;7:105-12.

7 MacMahon S, Collins G, Rautahariu P, et al. Electrocardiographic left ventricular hypertrophy and effects of antihypertensive drug therapy in hypertensive participants in the multiple risk factor intervention trial.
parte of participants in the multiple risk

8 Van Hoof R. Left ventricular hypertrophy in elderly hypertensive patients: a report from the European Workin Party on high blood pressure in the elderly trial. $\mathrm{Am}$ Med 1991;90(suppl 3A):55S-59S

9 Reichek N, Franklin BB, Chandler T, Muhammad A Plappert T, St John Sutton M. Reversal of left ventricular hypertrophy by antihypertensive therapy. Eur Heart 1982;3:165-9.

10 Cherchi A, Sau F, Seguro C. Regression of left ventricular hypertrophy after treatment of hypertension by chlorthalidone for one year and other diuretics for two years. $\mathcal{f}$ Hypertens 1983;(suppl 2):278-80.

11 Ferrara LA, de Simone G, Mancini M, Fasano ML, Pasanisi $F$, Vallone $G$. Changes in left ventricular mass during a double-blind study with chlorthalidone and slow-release nifedipine. Eur $\mathcal{f}$ Clin Pharmacol 1984;27: 525-8.

12 Ferrara LA, de Simone G, Pasanisi F, Mancini M. Left ventricular mass reduction during salt depletion in arterventricular mass reduction during salt depletion
ial hypertension. Hypertension 1984;6:755-9.

13 Mace PJE, Littler WA, Glover DR, Rowlands DB, Stallard TJ. Regression of left ventricular hypertrophy in hypertension: comparative effects of three different drugs. $f$ Cardiovasc Pharmacol 1985; 7:S52-5.

14 Sami M, Haichin R. Regression of left ventricular hypertrophy in hypertension with indapamide. Am Heart $f$ 1991;122:1215-8.

15 Komajda M, Klimczak K, Boutin B, Brackman F, Guez $D$, Grosgogeat Y. Effects of indapamide on left ventricular mass and function in systemic hypertension with left ventricular hypertrophy. Am $\mathcal{f}$ Cardiol 1990;65: $37 \mathrm{H}-42 \mathrm{H}$.

16 Gottdiener J, Reda D, Notargiacomo A, Materson B. Comparison of monotherapy effects on $\mathrm{LV}$ mass regression in mild-to-moderate hypertension: differences between short and long-term therapy [abstract]. $\exists \mathrm{Am}$ between short and long-term the
Coll Cardiol 1992;19(suppl A):85.

17 Devereux RB, Savage DD, Sachs I, Laragh JH. Effect of blood pressure control on left ventricular hypertrophy blood pressure control on left ventricular hypertrophy (suppl III):111-36.
18 Drayer JIM, Gardin JM, Weber MA, Aronow WS Changes in ventricular septal thickness during diuretic therapy. Clin Pharmacol Ther 1982;32:283-8.

19 Wollam GL, Hall WD, Porter VD, et al. Time course of regression of left ventricular hypertrophy in treated hypertensive patients. $A m \mathcal{F}$ Med 1983;75(suppl 3A): 100-10.

20 Giles TD, Sander GE, Roffidal LC, et al. Comparison of nitrendipine and hydrochlorothiazide for systemic hypertension. Am $f$ Cardiol 1987;60:103-6.

21 Messerli FH, Nunez BD, Nunez MM, et al. Hypertension and sudden death: disparate effects of calcium entry blocker and diuretic therapy on cardiac dysrhythmias. Arch Intern Med 1989;149:1262-7.

22 Langford HG, Blaufox MD, Borhani NO, Curb JD, et al. Is thiazide-produced uric acid elevation harmful? Analysi of data from the hypertension detection and follow-up program. Arch Intern Med 1987;147:645-9.

23 Prichard BNC, Owens CWI, Woolf AS. Adverse reaction to diuretics. Eur Heart f 1992;13(suppl G):96-103.

24 Ramsay LE, Yeo WW, Jackson PR. Diabetes, impaired glucose tolerance and insulin resistanc

25 Eur Heart f 1992;13(suppl G):68-71. on the plasma lipid profile. Eur Heart $\mathcal{f}$ 1992;13 (suppl on : $: 61-7$.

26 Singh BN, Hollenberg NK, Poole-Wilson PA, Robertson JIS. Diuretic-induced potassium and magnesium deficiency: relation to drug-induced QT prolongation, cardiac arrhythmias and sudden death. $\mathcal{F}$ Hypertens 1992 10:301-16.

27 Dargie HJ, Cleland JGF, Leckie BJ, et al. Relation of arrhythmias and electrolyte abnormalities to survival in patients with severe chronic heart failure. Circulation 1987;75(suppl IV)IV98-107.

28 Papademetriou V, Price M, Notargiacomo A, et al. Effect of diuretic therapy on ventricular arrhythmias in hypertensive patients with or without left ventricular hypertrophy. Am Heart $\mathcal{F}$ 1985;110:595-9.

29 Holland OB, Nixon JV, LaVon Kuhnert. Diuretic-induced ventricular ectopic activity. $A m \mathcal{f} M e d$ 1981;70:762-8.

30 Papademetriou V. Diuretics, hypokalemia, and cardiac arrhythmias: a critical analysis. Am Heart $\mathcal{f} 1986 ; 111$. 1217-24

31 Papademetriou V, Burris JF, Notargiacomo A, Fletcher $\mathrm{RD}$, Freis ED. Thiazide therapy is not a cause of arrhythmia in patients with systemic hypertension. Arch Intern Med 1988;148:1272-6.

32 Papademetriou V, Fletcher R, Khatri IM, Freis ED. Diuretic-induced hypokalemia in uncomplicated systemic hypertension: effect of plasma potassium correction on cardiac arrhythmias. Am $f$ Cardiol 1983;52: 1017-22.

33 Papademetriou V, Notargiacomo A, Heine D, Fletcher RD, Freis ED. Effects of diuretic therapy and exerciserelated arrhythmias in systemic hypertension. $A m \mathcal{F}$ Cardiol 1989;64:1152-6.

34 Madias JE, Madias NE, Gavras HP. Nonarrhythmogenicity of diuretic-induced hypokalemia. Arch Interm Med 1984;144:2171-6.

35 Collins R, Peto R, MacMahon S, Herbert P, Siebach NH, Eberlein KA, et al. Blood pressure, stroke and coronary heart disease. Part 2, short-term reductions in blood pressure: overview of randomised drug trials in their epidemicological context. Lancet 1990;335:827-38.

36 Multiple Risk Factor Intervention Trial Research Group. Risk factor changes and mortality results. $\mathfrak{F} A M A 1982$ 248: 1465-77.

37 Hypertension Detection and Follow-up Program Cooperative Group. Five-year findings of the hypertension detection and follow-up program. I. Reduction in mortality of persons with high blood pressure, including mild hypertension. $¥ A M A 1979 ; 242: 2562-77$.

38 Steward DE, Ikram H, Espiner EA, Nicholls MG Arrhythmogenic potential of diuretic induced hypokalaemia in patients with mild hypertension and hypokalaemia in patients with mild hypertension

39 Hollifield J, Slaton P. Thiazide diuretics, hypokalaemia and cardiac arrhythmias. Acta Medica Scandinavica and cardiac arrhythmias.

40 Hollifield J. Thiazide treatment of systemic hypertension effects on serum magnesium and ventricular ectopic activity. Am $\mathcal{f}$ Cardiol 1989;63:22G-25G.

41 Hollifield JW. Thiazide treatment of hypertension: effect of thiazide diuretics on serum potassium, magnesium and ventricular ectopy. $A m \mathcal{F} \mathrm{Med} 1986 ; 80$ (suppl $4 \mathrm{~A}$ ) 8-12.

42 Freis E. The cardiotoxicity of thiazide diuretics: review of the evidence. 7 Hypertens 1990;8 (suppl 2):S23-32.

43 Amery A, Birkenhager W, Brixko P, Bulpitt C, Clement $\mathrm{D}$, de Ruyttere $\mathrm{M}$, et al. Mortality and morbidity results from the European Working Party on high blood pressure in the elderly trial. Lancet 1985;i:1349-54.

44 Dahlöf B, Lindholm L, Hansson L, Schersten B, Ekbom T, Wester PO. Morbidity and mortality in the Swedish T, Wester PO. Morbidity and mortality in the Swedish trial in old patients with hypertensic

45 SHEP Cooperative Research Group. Prevention of stroke by antihypertensive drug treatment in older persons with by antihypertensive drug treatment in older persons with
isolated systolic hypertension. $f A M A \quad 1991 ; 285$ 3255-64. 